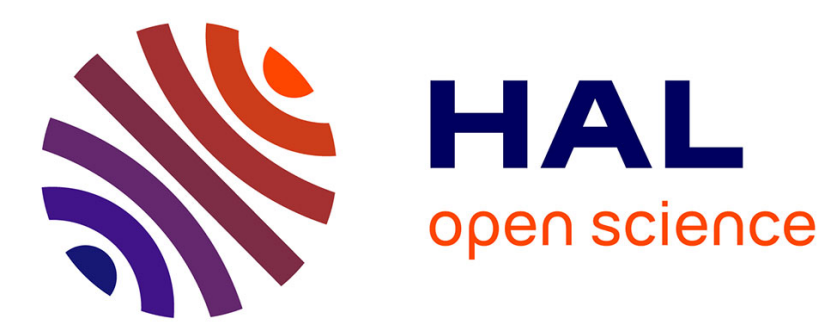

\title{
Non-Fickian dispersion in porous media: 2. Model validation from measurements at different scales
}

Tanguy Le Borgne, Philippe Gouze

\section{To cite this version:}

Tanguy Le Borgne, Philippe Gouze. Non-Fickian dispersion in porous media: 2. Model validation from measurements at different scales. Water Resources Research, 2008, 44, pp.W06427. 10.1029/2007WR006279 . insu-00373698

\section{HAL Id: insu-00373698 \\ https://hal-insu.archives-ouvertes.fr/insu-00373698}

Submitted on 29 Jun 2016

HAL is a multi-disciplinary open access archive for the deposit and dissemination of scientific research documents, whether they are published or not. The documents may come from teaching and research institutions in France or abroad, or from public or private research centers.
L'archive ouverte pluridisciplinaire HAL, est destinée au dépôt et à la diffusion de documents scientifiques de niveau recherche, publiés ou non, émanant des établissements d'enseignement et de recherche français ou étrangers, des laboratoires publics ou privés. 


\title{
Non-Fickian dispersion in porous media: 2. Model validation from measurements at different scales
}

\author{
T. Le Borgne $e^{1,2}$ and P. Gouze ${ }^{1}$ \\ Received 18 June 2007; revised 20 February 2008; accepted 20 March 2008; published 28 June 2008.
}

[1] We aim at testing and validating a mobile-immobile mass transfer model from a set of single-well injection withdrawal tracer tests in a heterogeneous porous aquifer. By varying the duration of the injection phase, different volumes of aquifer are investigated by the tracer. Hence, we focus the transport model validation not only on reproducing a single breakthrough curve (BTC) but also on the model's capacity to predict the amount of mixing as a function of the volume visited by the tracer. All the BTCs are strongly asymmetric, as expected when dispersion is controlled by diffusive mass transfers between the mobile water and the immobile water part of the porosity. However, the BTC cannot be modeled by a conventional mobile-immobile mass transfer model with a simple power law memory function. To account for that, we implement a continuous time random walk model in which the transition time distribution $\psi(t)$, which is related to the excursion time probability of the tracer in the immobile domain, is a dual-slope power law distribution. The model best fits the BTC data set with a transitional regime controlled by $\psi(t) \sim t^{-2}$ and an asymptotic regime characteristic of the conventional double-porosity model with $\psi(t) \sim t^{-1.5}$. This work emphasizes that high-resolution concentration measurement and multiple-scale tracer tests are required for assessing solute dispersion models in heterogeneous reservoirs and for subsequently obtaining reliable predictions.

Citation: Le Borgne, T., and P. Gouze (2008), Non-Fickian dispersion in porous media: 2. Model validation from measurements at different scales, Water Resour. Res., 44, W06427, doi:10.1029/2007WR006279.

\section{Introduction}

[2] The prediction of the fate of dissolved species, such as pollutants, in the subsurface requires the definition and calibration of solute transport models. How to represent appropriately the relation between heterogeneous geological structures and the solute transport properties is a highly debated issue [Harvey and Gorelick, 2000; Benson et al., 2001; Berkowitz et al., 2002; Le Borgne et al., 2007]. Several tracer tests, both at field scale [Adams and Gelhar, 1992; Meigs and Beauheim, 2001; Becker and Shapiro, 2003] and laboratory scale [Silliman and Simpson, 1987; Levy and Berkowitz, 2003], show that the classical advection-dispersion equation (ADE) is not valid to represent solute transport in heterogeneous reservoirs because it underestimates, often strongly, the effective solute residence times. However, there is rarely enough data to validate alternative transport models, that is, to demonstrate that a given model and the parameters fitted with a given tracer test can be used to predict dispersion at other scales, or for different experimental conditions. In general, equally good fits of a single breakthrough curve (BTC) may be obtained from different conceptual models [Sánchez-Vila

\footnotetext{
${ }^{1}$ Géosciences, UMR 5243, Université de Montpellier 2, CNRS, Montpellier, France.

${ }^{2}$ Now at Géosciences Rennes, UMR 6118, Université de Rennes 1, CNRS, Rennes, France.
}

Copyright 2008 by the American Geophysical Union. 0043-1397/08/2007WR006279 and Carrera, 1993]. One of the major tasks in hydrogeology is therefore to design field methods that allow validating transport models and measure site specific parameters.

[3] One way to improve the uniqueness of the tracer test interpretation is to stress the system in different configurations. Meigs and Beauhein [2001] and Becker and Shapiro [2003] performed both single-well injection withdrawal (SWIW) and well-to-well tracer tests to study transport in heterogeneous aquifers. A major concern with well-to-well tracer tests, in addition to the necessity of drilling several wells to investigate scale effects, is the poor recovery of tracer mass that is often observed, especially in highly heterogeneous media. Indeed, the probability of retrieving the injected tracer mass decreases rapidly with the well separation distance because of the complex threedimensional patterns of the flow paths. SWIW tracer tests, also called push-pull or echo tracer tests, are performed by injecting a given mass of tracer in the medium and reversing flow after a certain time in order to measure the tracer breakthrough curve at the injection point [Gelhar and Collins, 1971; Magnico et al., 1993; Tsang, 1995; Haggerty et al., 2001; Khrapitchev and Callaghan, 2003] (Figure 1). This technique presents several advantages. First, the reversal of flow leads to an optimal tracer mass recovery. Second, it is expected that the effect of large-scale heterogeneities is canceled because of the reversal of the flow field [Becker and Shapiro, 2003; Khrapitchev and Callaghan, 2003; Gouze et al., 2008]. Thus, the dispersion measured from breakthrough curves is only caused by tracer molecules that 
INJECTION WITHDRAWAL

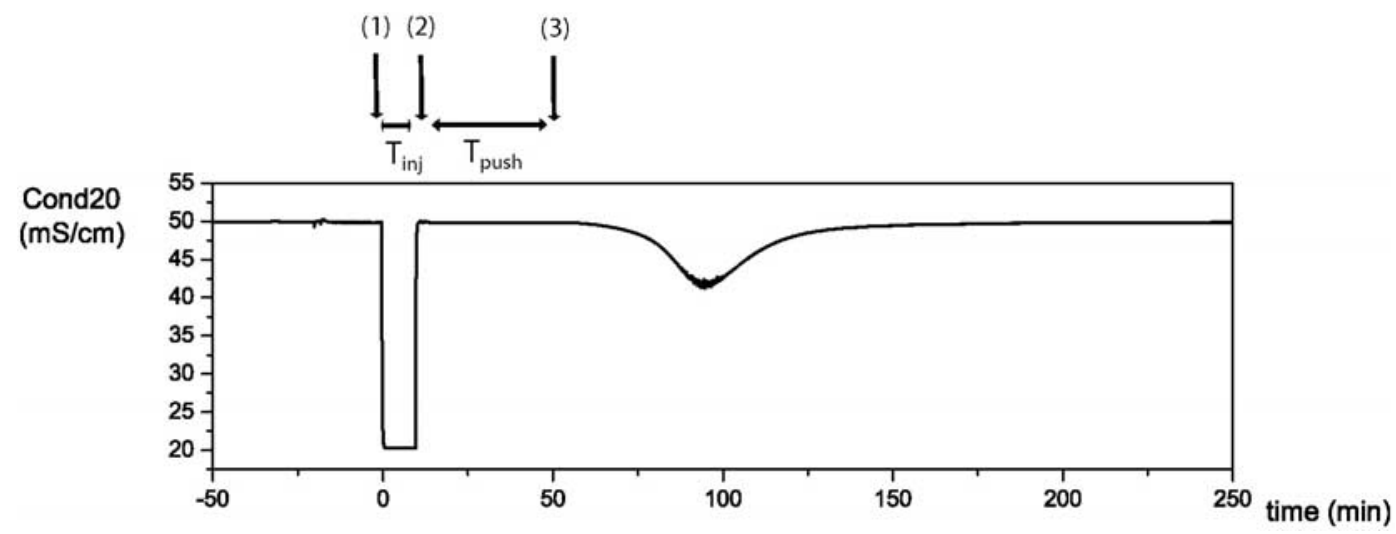

Figure 1. Example of SWIW tracer test monitoring (injection of water of conductivity $20 \mathrm{mS} / \mathrm{cm} \mathrm{in} \mathrm{the}$ reservoir saturated with water of conductivity $50 \mathrm{mS} \mathrm{cm}^{-1}$. All the tracer tests are performed at constant flow rate $\mathrm{Q}= \pm 12 \mathrm{~L} \mathrm{~min}^{-1}$. Tracer injection starts at the point marked (1), tracer injection stops and flushing fluid injection starts at the point marked (2), and the pump is reversed at the point marked (3).

do not follow the same path on the injection and the withdrawal phases. Consequently, the measured dispersion represents mixing caused by irreversible processes such as diffusion but not the advective spreading caused by largescale heterogeneities. In particular, such tests are well suited to investigate mobile-immobile (MIM) mass transfer processes or matrix diffusion [Tsang, 1995]. Finally, the tracer may be pushed at different distances from the injection point, thus visiting different volumes of the system using a single well. This latter aspect, rarely investigated in previous studies, is the focus of the present study.

[4] The few SWIW tracer tests published recently show a very long time for tracer recovery characterized by power law BTC tailing [Meigs and Beauheim, 2001; Becker and Shapiro, 2003]. However, the origin of the fitted power law exponents, and more generally of the non-Fickian dispersion, is debated [Haggerty et al., 2001]. Becker and Shapiro [2003] implemented a multichannel model to fit a single SWIW tracer test BTC. Alternatively, Haggerty et al. [2001] fitted their SWIW tracer test BTCs using a mobileimmobile (MIM) mass transfer model in which the nonFickian dispersion arises from small-scale diffusion and mixing. MIM mass transfer models assume that solutes can be trapped in immobile zones from which they are slowly released by diffusion [Haggerty and Gorelick, 1995].

[5] Whatever the model used to interpret the data, a critical point is whether the model can be used to predict dispersion at different scales. For instance, the power law behavior, characterizing heavy tailed breakthrough curves, may be valid over a range of scales only [Dentz et al., 2004]. This issue is specifically addressed in the present paper by analyzing SWIW tracer tests with a large range of injection durations. The primary objective of this paper is thus to investigate the relevance of a unique dispersion model to represent the whole data set. A particle-tracking random walk formulation, based on the continuous time random walk approach [Scher and Lax, 1973; Berkowitz et $a l ., 2006]$, is proposed (section 3) and then parameterized to reproduce the tracer test BTCs (sections 4). In section 5, the validation of the transport model from measurements at different scales is discussed and the main conclusions are provided.

\section{SWIW Tracer Test Data Set}

[6] The SWIW tracer tests data set analyzed in the present study is presented in details in Gouze et al. [2008]. The tracer tests were conducted in a paleoreef porous reservoir displaying important small-scale heterogeneity, but no large-scale discontinuities or measurable permeability heterogeneity [Gouze et al., 2008]. The experimental site is situated $6 \mathrm{~km}$ inland at about $50 \mathrm{~km}$ south from the city of Palma de Majorca, Balearic Islands. At the site location, seawater intrusion is observed at a depth of $62 \mathrm{~m}$ with a transition from continental water to seawater of about $16 \mathrm{~m}$. The tracer tests were performed in the seawater intrusion zone at depth $94 \mathrm{~m}$. In this zone, the rocks belong to the reef distal talus which is quite homogeneous at meter to decameter scale. The total porosity of the reservoir at depth $94 \mathrm{~m}$ is $43.5 \pm 1.5 \%$ (measured on 2 to $10 \mathrm{~cm}^{3}$ samples). The mobile porosity (i.e., porosity forming the permeability of the rock) was evaluated at $38 \%$ from tracer tests performed using a borehole core of length $720 \mathrm{~mm}$ (diameter $90 \mathrm{~mm}$ ). This ratio between the total porosity and the mobile (or advection) porosity was confirmed by measurements on X-ray microtomography images (resolution $5.4 \mu \mathrm{m}$ ). The microscale heterogeneity including the micropores and the dead-end clusters that form the immobile domain can be although individualized from processing high-resolution (e.g., resolution $1 \mu \mathrm{m}$ ) X-ray microtomography [see Gouze et al., 2008, Figures 3 and 5]. The connected micropore structure appears very tortuous with high proportion of dead ends were diffusion dominant transport is expected. The tortuosity and the large specific area of the mobile-immobile interface is also promoting large excursion probability of the tracer molecules in the immobile zones.

[7] The value of the data set discussed here, compared to other published results, is threefold. First, the experimental protocol allowed very short rectangle shaped tracer injection pulse (mimicking a Dirac injection), precisely controlled constant flow rate, and high-resolution continuous concentration recording directly in the interpacker chamber, reduc- 


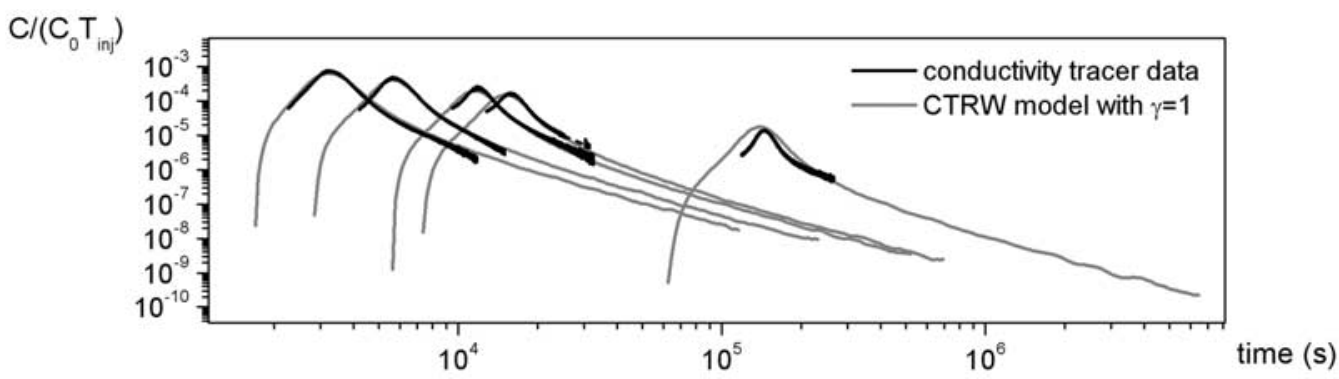

Figure 2. Comparison of tracer data (black lines) and model prediction (gray lines) at different scales for the conductivity data set. The parameters used in the model with $\gamma=1$ are $\alpha=10^{-3}$ and $A=0.25$.

ing considerably the dispersion in the equipment itself. Second, the authors used a combination of two tracers, for which measurement techniques are distinct (conductivity measurement and optical measurement), in order to verify that the tracers behave as passive tracers and that density effects do not induce perturbations on the BTCs. Third, distinctly different volumes of rock are investigated by increasing the water flushing injection in order to tackle time and space-scale effects. For the present experiments, the maximum volume sampled by the tracer is

$$
V_{\max }=\frac{Q t_{i n j}}{n}-h \pi r_{w}^{2}
$$

where $Q$ is the injection rate, $n$ is the average effective porosity and $r_{\mathrm{w}}$ is the well radius. Assuming an effective porosity equal to $30 \%$, the visited volume of the SWIW tracer tests ranges from 0.8 to $45 \mathrm{~m}^{3}$ for injection duration ranging from $20 \mathrm{~min}$ to $19 \mathrm{~h}$, respectively. Two kinds of tracers were used. The first tracer (tracer 1) is fresh water injected in the formation saltwater. Its concentration is measured with a conductivity probe (allowing a 3 order of magnitude range of concentration measurements). The second tracer is uranine (tracer 2). Its concentration is measured using a newly developed high-resolution sensor, called TeLog, allowing a 5 order of magnitude range of concentration measurements.

[8] The breakthrough curves studied here are heavy tailed, indicating a very large tracer residence time distribution (Figure 2). In the present case, we anticipate that dispersion is controlled mainly by irreversible processes such as diffusion into immobile zones and mixing and not so much by large-scale spreading and channeling. Borehole wall images and geophysical parameters profiles show that the rock is relatively homogeneous over the tested interval, and none of the observed structures are expected to act as immobile zones at this scale. However, at microscale, the tortuosity of the microporosity and the submillimeter size dead ends and unconnected pore clusters are expected to act as immobile zones [Gouze et al., 2008]. The large surfaceto-volume ratio of the microporous phase implies that solute can be repeatedly trapped by diffusion and thus move macroscopically slowly through the medium.

\section{Particle-Tracking Formulation of a CTRW Transport Model for Cylindrical Divergent/ Convergent Flow}

[9] As a result of the analysis of the structural heterogeneities from micrometric to metric scale over the tested interval, we focus on a MIM mass transfer model to fit the tracer test data. In the following, we derive a CTRW model for cylindrical divergent/convergent flow, which is formally equivalent to a MIM mass transfer model [Dentz and Berkowitz, 2003].

[10] Considering MIM mass transfers, transport is modeled by the advection-dispersion equation in the mobile zone and a source term $F_{\mathrm{m}}$ representing mass transfer between mobile and immobile zones [Carrera et al. 1998; Haggerty et al., 2001; Schumer et al., 2003]:

$$
\frac{\partial c}{\partial t}=\nabla(D \nabla c)-v \nabla c-F_{m}
$$

where $c$ is the tracer concentration in the mobile fluid, $D$ is the dispersion tensor and $v$ is the mean pore velocity. For linear mass exchange processes, such as diffusion, the source term may be expressed as a convolution product of the concentration variations in the mobile zone with a "memory function, "which represents the mass flux to the immobile zones per unit volume of aquifer, for a unit change in concentration in the mobile zone [Carrera et al., 1998; Haggerty et al., 2000]. The formulation of this term depends on the geometry of immobile zones and on the variability of mass transfer or diffusion rates [Haggerty et al., 2000]. In some cases, analytical solutions of (2) can be derived in Laplace space. However, in the case of SWIW tracer tests, the analytical solutions may not be easily obtained since the withdrawal phase starts with an initial distribution of concentration in the mobile and immobile phase that cannot be implemented easily in the Laplace domain. Other numerical methods to solve equation (2) are presented by Carrera et al. [1998]. Here, we use a random walk particle-tracking approach [Tsang and Tsang, 2001; Dentz et al., 2004]. Following this approach, which corresponds to the conceptual framework of CTRW, the solute concentration distribution in space and time is represented by the probability density functions of random walkers performing random steps $\Delta \mathrm{r}$ in random times $\Delta \mathrm{t}$ so that the radial position and time between increments $n$ and $n$ +1 are updated as follows:

$$
\left\{\begin{array}{l}
r_{n+1}=r_{n}+\Delta r \\
t_{n+1}=t_{n}+\Delta t .
\end{array}\right.
$$

[11] Generally, particle-tracking approaches use a fixed time increment $\Delta t$ and a spatial increment $\Delta r$ drawn from a 
Gaussian distribution [e.g., Kinzelbach, 1988]. The retention of solutes in the immobile phase can be represented by considering a time increment stochastically drawn from a waiting time distribution [Tsang and Tsang, 2001; Dentz et al., 2004]. The choice of the waiting time distribution depends on the mass transfer process under consideration [Dentz et al., 2004].

[12] For tracer injection between packers in heterogeneous porous media, the flow field is dominantly cylindrical in the vicinity of the well and tends progressively to a spherical geometry further from the well. We tested both cylindrical and spherical geometries and found only slight differences in the breakthrough curve shapes using the characteristics of the SWIW tracer tests under consideration. The flow field geometry is found to influence mainly the part of the curve that is controlled by the Fickian dispersion. Conversely, most of the curve, including the power law part, does not depend on the geometry. Hence, a cylindrical geometry is assumed in the remainder of the paper. We expect that this choice would introduce minor uncertainties on the estimation of the dispersivity but would not influence the estimation of the waiting time distribution.

[13] We start with the definition of a random walk particle-tracking model for solving the ADE in radial coordinates. In cylindrical geometry, the formulation of the $\mathrm{ADE}$ for the solute concentration $c$ is as follows:

$$
\frac{\partial c}{\partial t}=-\frac{k_{v}}{r} \frac{\partial c}{\partial r}+\frac{\alpha k_{v}}{r} \frac{\partial^{2} c}{\partial r^{2}},
$$

where $\alpha$ is the dispersivity, assumed constant over the domain and $k_{v}=Q / 2 \pi h n$, with $Q$ is the flow rate, $n$ is the porosity and $h$ is the height of the injection interval. The term $k_{v} / r$ in (3) denotes the averaged velocity $\langle v\rangle$ at a given radial position $r$. Note that, for a spherical geometry $\langle v\rangle=$ $k_{v} / r^{2}$ with $k_{v}=Q / 4 \pi n$.

[14] The random walk approach used to solve the ADE is based on the similarity of the ADE solution and the FokkerPlanck equation solution [e.g., Delay et al., 2005], where the Fokker-Planck equation is

$$
\frac{\partial p}{\partial t}=-\frac{\partial\left(L_{1} p\right)}{\partial r}+\frac{\partial^{2}\left(L_{2} p\right)}{\partial r^{2}}
$$

with $p$ the probability density under consideration, $L_{1}$ the drift coefficient and $L_{2}$ the dispersion coefficient. The similarity between equations (4) and (5) is obtained by the following change of variable: $m=r c$. Then, because $k_{v}$ and $\alpha$ are constants, equation (4) becomes

$$
\frac{\partial m}{\partial t}=-\frac{\partial \frac{k_{v}}{r} m}{\partial r}+\frac{\partial^{2} \frac{\alpha k_{v}}{r} m}{\partial r^{2}},
$$

with $L_{1}=k_{v} / r$ and $L_{2}=\alpha k_{v} / r$. Under this form, the equation may be solved by the following random walk procedure [Delay et al., 2005]:

$$
\left\{\begin{array}{l}
t_{n+1}=t_{n}+\Delta t \\
r_{n+1}=r_{n}+<v>\Delta t+\xi(\Delta t)
\end{array}\right.
$$

and $\xi(\Delta t)$ is a zero mean random Gaussian increment defined by its variance $\left\langle\xi^{2}(\Delta t)\right\rangle=2 D(r) \Delta t$ where $D(r)=$ $\alpha k_{v} / r$ is the dispersion coefficient and $\Delta t$ is the discretization time. To model SWIW tracer tests, the flow is reversed at time $\mathrm{t}=T_{\text {push }}+T_{\text {inj }}$ (see Figure 1 ). Particle movements are tracked until all particles are back to the injection point. The result of the simulation is the relative concentration at the well:

$$
c\left(t, r=r_{w}\right) / c\left(0, r=r_{w}\right)=m\left(t, r=r_{w}\right) / m\left(0, r=r_{w}\right) .
$$

[15] To account for mass exchange with immobile zones we must add a random time increment $\tau$ at each time step [Tsang and Tsang, 2001; Dentz and Berkowitz, 2003]. Thus, the formulation of the random walk procedure is as follows:

$$
\left\{\begin{array}{l}
t_{n+1}=t_{n}+\Delta t+\tau(\Delta t) \\
r_{n+1}=r_{n}+<v>\Delta t+\xi(\Delta t) .
\end{array}\right.
$$

[16] The key property that controls the breakthrough curve shape is the asymptotic (late time) scaling of the waiting time distribution $\psi(\tau)$. A power law scaling can be chosen to represent heavy-tailed tracer breakthrough curves:

$$
\psi(\tau)=A(\tau / \Delta t)^{-1-\gamma} .
$$

[17] The model proposed is basically a CTRW model, defined by a joint transition length and time distribution (equation (8)), which is equivalent to the MIM mass transfer model given by equation (2). This equivalence has been demonstrated by Dentz and Berkowitz. [2003]. The source term in $F_{\mathrm{m}}(2)$ is expressed as the following convolution product:

$$
F_{m}=g_{t}^{*} \frac{\partial C}{\partial t}=\int_{0}^{\infty} g_{t}(\tau) \frac{\partial C}{\partial t}(t-\tau) d \tau
$$

where the memory function in the Laplace space $\left(g^{*}\right)$ is given by

$$
g^{*}(s)=\frac{1-\psi^{*}(s)(1+s \Delta t)}{s \Delta t \psi^{*}(s)},
$$

where $s$ is the Laplace variable and $\psi^{*}$ is the Laplace transform of $\psi$.

[18] The memory function is embedding the distribution of waiting times in the immobile zone. A power law distribution of retention times (equation (9)) is equivalent to a power law memory function $g(t)$ [Dentz and Berkowitz, 2003]:

$$
g(t)=B t^{-\gamma}
$$

[19] Statistically pertinent simulations require $10^{6}$ to $10^{7}$ particles. The simulation results are independent of the discretization time $\Delta t$, provided it is very small compared to the simulation time. For all the simulations, we used $\Delta t=1 \mathrm{~s}$. 


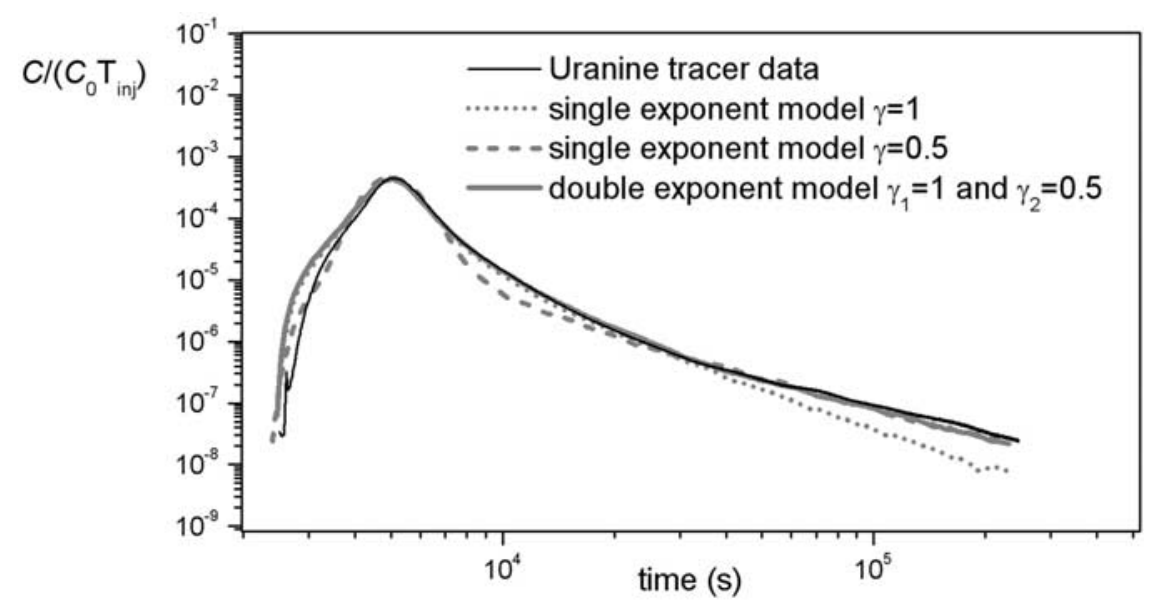

Figure 3. Breakthrough curve obtained using uranine as a tracer and the CTRW model with a single exponent $\gamma=1$, the CTRW model with a single exponent $\gamma=0.5$ (equivalent to the classical doubleporosity model), and the CTRW model with two characteristic exponents $\left(\gamma_{1}=1\right.$ and $\left.\gamma_{2}=0.5\right)$. The experiment is performed by injecting 20 ppm mass of $99 \%$ pure uranine diluted in the formation water. The tracer injection time is $\Delta t=2 \mathrm{~min}$, and the injection duration is $T_{p u s h}=40 \mathrm{~min}$.

[20] The single slope model (equation (9)) contains three parameters: the dispersivity $\alpha$ (Fickian dispersion term), the exponent $\gamma$ of the waiting time distribution, and its proportionality coefficient $A$ (equation (9)). These parameters can be interpreted in the MIM mass transfer conceptual framework as follows. The dispersivity controls the dispersion properties in the mobile domain. The exponent $\gamma$ controls the scaling of the retention time distribution in the immobile zone. An exponent $\gamma=0.5$ corresponds to the classical double-porosity model [Carrera et al., 1998]. For $\gamma>2$, the classical advection-dispersion equation is recovered. An exponent $\gamma \neq 0.5$ is expected when the immobile zones display a distribution of characteristic sizes (e.g., distribution of spheres) or if the effective diffusion coefficient in the immobile zone is spatially variable [Haggerty et al., 2001]. The coefficient $A$ (equation (9)) is related to the coefficient $B$ of the memory function (equation (12) through equation (11)). From Dentz and Berkowitz [2003, equation (D9)], we derive the following simple relationship:

$$
B=\frac{A}{\Delta t} .
$$

[21] We validated this random walk approach by comparison with the TRANSIN model [Carrera et al., 1998]. The advantage of the random walk approach is that it can handle small dispersivity coefficients, such as needed for the present study, without numerical instability problems.

\section{Breakthrough Curve Modeling}

\subsection{Conductivity Breakthrough Curves}

[22] A set of parameters $(\alpha, \gamma, A)$ can be obtained from fitting the CTRW model to a single breakthrough curve. The exponent $\gamma$ controls the late time decrease of the BTC tails. For such model, the late time concentration scales as $c(t) \propto t^{-1-\gamma}$ [Haggerty et al., 2001]. The resolution of the conductivity probe does not allow the measurement of tracer concentration over sufficient timescales to observe power law scaling. Over the period of observation, the evolution of late time concentration with time is not a power law. However, an optimal exponent $\gamma$ can be relatively well defined from the fit of the preasymptotic temporal evolution of concentration as illustrated in Figure 2. The dispersivity $\alpha$ controls the spreading of the BTC in the vicinity of the peak. However, as the duration of the tracer test increases, the width of the BTC becomes more and more insensitive to this parameter. For the longest-term tracer tests, the BTC shape is entirely controlled by the waiting time distribution. Moreover, in the case of SWIW tracer tests, the dispersivity and the effective porosity cannot be estimated independently since the mean advective velocity is poorly constrained [Becker and Shapiro, 2003]. To estimate $\alpha$ from the tracer tests, the effective porosity is set to 0.3 (corresponding to the value measured on cores). The coefficient $A$ controls the proportion of the curve that is affected by the slow release of solutes from immobile zones. Figure 2 shows an example of the best fit of a BTC by the CTRW model. The optimal set of parameters in this example is $\gamma=1, \alpha=10^{-3} \mathrm{~m}$ and $A=0.25$. Similarly to previously published SWIW tracer tests [e.g., Haggerty et al., 2001], we obtain an exponent different from the classical double-porosity model (i.e., $\gamma=$ $0.5)$.

[23] The proposed CTRW model is found to model well the conductivity breakthrough curves for different push durations with a single set of parameters $(\alpha, \gamma, A)$. All the modeled breakthrough curves in Figure 2 are obtained with the same parameters. The predictions of the model, that was fitted only for the experiment with $T_{\text {push }}=40 \mathrm{~min}$, are very good. The main discrepancy between the model and the data is found for the longest-term test. The tracer breakthrough curve has less spread than predicted by the model. In addition, the tracer breakthrough curve peak is lower than predicted by the model. This tracer test, for which experimental bias is anticipated, requires specific attention that will be discussed in section 4.4 .

[24] The calculated dispersion coefficient $\alpha=10^{-3} \mathrm{~m}$ is relatively small, but consistent with small size of the heterogeneities in the rock [Gouze et al., 2008]. Statistical analyses of the pore structure from microtomography 


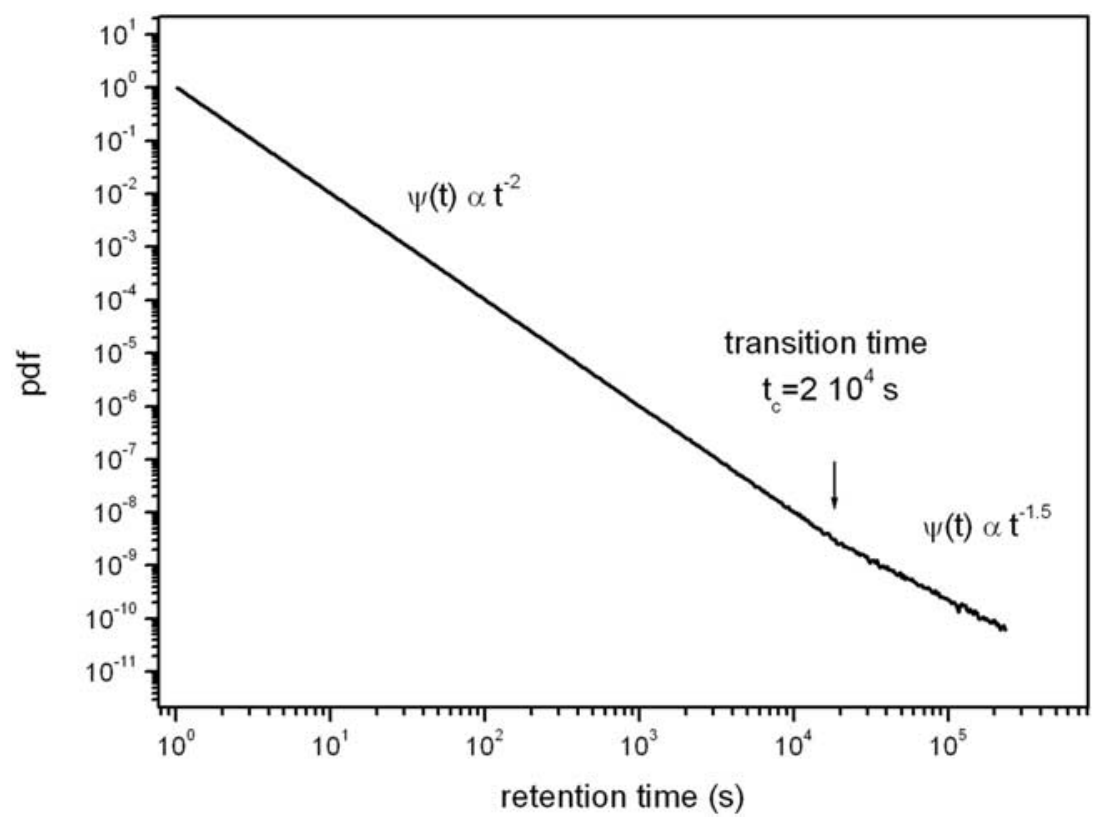

Figure 4. Transition time distribution for the CTRW model (equation (8)) with two characteristic exponents $\left(\gamma_{1}=1\right.$ and $\left.\gamma_{2}=0.5\right)$.

images (using the lineal path function for example; see Torquato [2001]) show a decorrelation length of the porosity structures of less than $100 \mu \mathrm{m}$, which is consistent with the value that we obtain for the dispersivity. Such a small value is also consistent with the fact that the effect of largescale heterogeneities is expected to be canceled in SWIW tracer tests because of the reversal of the flow field.

\subsection{Fluorescent Dye (Uranine) Breakthrough Curves}

[25] The uranine breakthrough tests allow investigating a larger concentration range than the conductivity tests (gain of 2 orders of magnitude in concentration). A good agreement is obtained between both tests [Gouze et al., 2008, Figure 11]. However, the CTRW model with $\gamma_{1}=1$, fitted from the conductivity tests, is not able to model the late time part of the uranine BTCs (Figure 3). The CTRW model with $\gamma_{1}=1$ appears to be consistent up to a characteristic time where the breakthrough curve deviates from the model. This effect is not seen for conductivity breakthrough curves because the sensor resolution does not allow investigation of this regime. To model this unconventional behavior, we tested a CTRW model with two characteristic exponents, such that the transition time distribution scales as $t^{-1-\gamma_{1}}$ for $t<t_{\mathrm{c}}$ and $t^{-1-\gamma_{2}}$ for $t>t_{\mathrm{c}}$, where $t_{\mathrm{c}}$ is a time that characterizes the transition between the two regimes (Figure 4).

[26] From the fit of the 2-2 uranine tracer test, we find that a transition between a regime $\gamma_{1}=1$ and $\gamma_{2}=0.5$ with a characteristic transition time $t_{c}=210^{4} \mathrm{~s}$ allows a good fit to this breakthrough curve (Figure 3). Note that the CTRW model with a single exponent $\gamma=0.5$ is not able to model the full breakthrough curve behavior (Figure 3). Figure 5 compares the prediction of this model for the two uranine tracer tests. The model that was fitted from tracer test 2-1

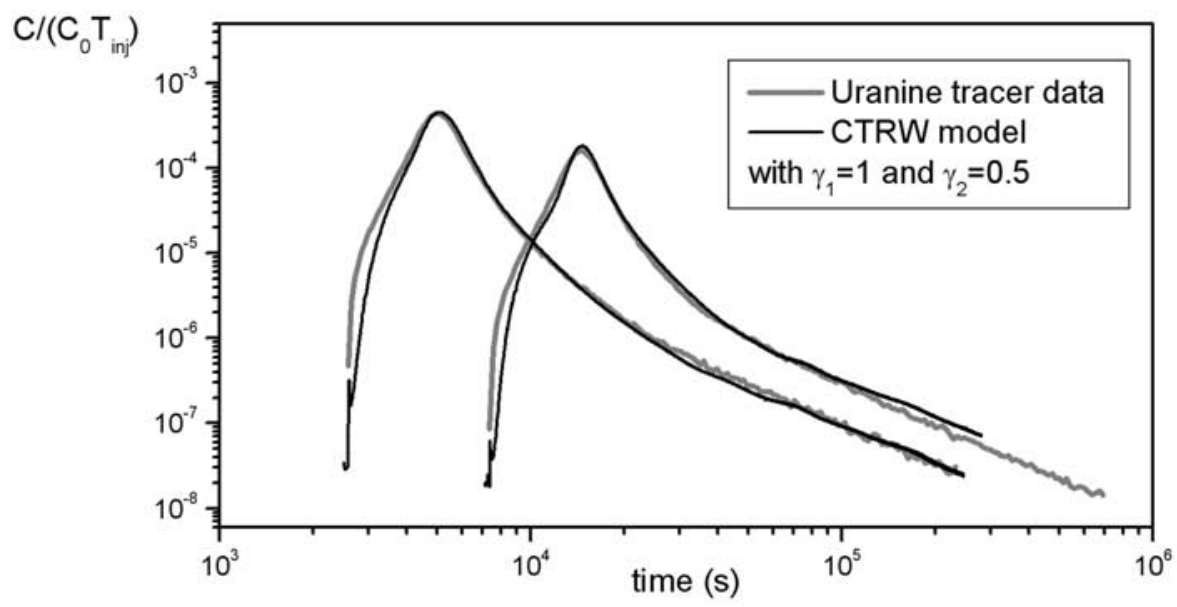

Figure 5. Comparison of tracer data and model prediction at different scales for the uranine tracer data set. The model used is the one defined in Figure $3\left(\gamma_{1}=1\right.$ and $\left.\gamma_{2}=0.5\right)$. 


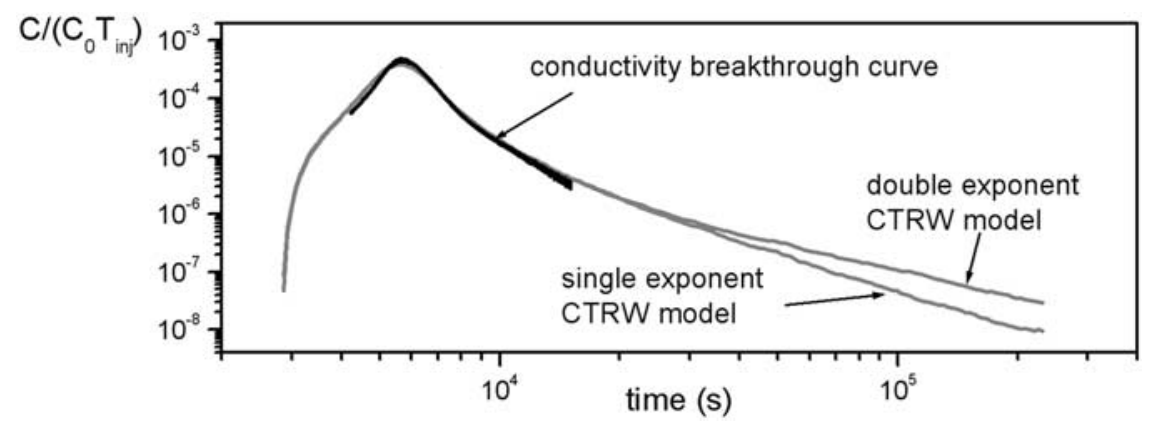

Figure 6. Comparison of the single-exponent model $(\gamma=1)$ and the two-exponent model $\left(\gamma_{1}=1\right.$ and $\left.\gamma_{2}=0.5\right)$ prediction for the trace $1-3$ experiment (gray curves). The trace $1-3$ breakthrough curve is shown as a black curve.

gives good prediction for tracer test 2-2 that has a push time that is three time that of tracer test $2-1$. Note also that the two-slope model is identical to the single exponent $\gamma=0.5$ model over the range of measurement of the conductivity breakthrough curves (Figure 6). Thus the two-exponent model is consistent with the whole tracer data set.

\subsection{Tracer Dilution and Mixing at Different Scales}

[27] As mentioned earlier, the breakthrough curves are expected to measure only mixing and not spreading, in the case of SWIW tracer tests. As a result, the breakthrough curve peak concentration is a measure of mixing, and is inversely proportional to the dilution index defined by Kitanidis [1994]. For the case of a one-dimensional transport for a simple advection-dispersion model, the peak concentration is expected to follow a power law of the total mean travel time, with an exponent -0.5 [Kitanidis, 1994]. More generally, in the case of a one-dimensional transport model based on Levy motion, the peak concentration is also expected to follow a power law of exponent $-1 / \alpha$, where $\alpha$ is the characteristic exponent of the alpha-stable velocity distribution [Benson et al., 2001] ( $\alpha=2$ for a Gaussian distribution). Yet, the change of the maximum concentration as a function of the mean travel time has not been investigated for SWIW tracer tests with MIM mass transfer in cylindrical geometry. Analyzing a set of numerical simulations, we find that the SWIW breakthrough curve peak concentration scales as a power law of the push time (Figure 7):

$$
c_{\max } \propto\left(2 T_{\text {push }}+T_{\text {inj }}\right)^{-\alpha} .
$$

[28] We found that the exponent $\alpha$ depends only on the exponent $\gamma$. This dependence appears to be nonlinear. No simple expression has been derived yet. Equation (14) shows how MIM mass transfer processes influence the tracer concentration mixing at different scales. It also shows that the analysis of the peak concentration as a function of the push time can be used to characterize the exponent $\gamma$.

[29] Except for the longest injection experiment, the scaling of the peak concentration is consistent with a CTRW model with exponent $\gamma=1$ (Figure 5). Indeed, the waiting time distribution of $\log (\psi)-\log (t)$ slope $-2(\gamma=1)$ controls the peak concentration over a significant range of spatial and temporal scales. The second scaling of the retention time distribution $\left(\gamma_{2}=0.5\right.$ in Figure 4) influences the scaling of the peak concentration for the longer-term tests. Hence, for the largest injection time, the maximum concentration is significantly lower than predicted by the CTRW model with $\gamma=1$ but it is consistent with that predicted by the CTRW model with two exponent $\gamma_{1}=1$ and $\gamma_{2}=0.5$. The long-term experiment is a special case that we discuss in the next section. The CTRW model with two exponents is thus able to model the breakthrough curve shape and the solute mixing at different scales.

[30] Note that the scaling peak time $t_{\text {Cmax }}$, obtained from the numerical simulations, is $t_{\text {Cmax }} \propto 2 T_{\text {push }}+T_{\text {inj. }}$. This scaling is also obtained for the SWIW tracer tests [Gouze et al., 2008, Figure 13]. Thus, we found that, in the case of SWIW tracer tests, the scaling of the peak time is independent of the transport parameters and of the transport models (ADE or CTRW). It only depends on the experimental setup ( $\left.T_{\text {push }}, T_{\text {inj }}\right)$.

\subsection{Long-Term Tracer Test (Tracer 1)}

[31] The long-term SWIW conductivity test was performed for push duration of nearly $19 \mathrm{~h}$. For this experiment, there was significant amount of unrecovered mass $(45 \%)$. Simple geometrical considerations indicate that the

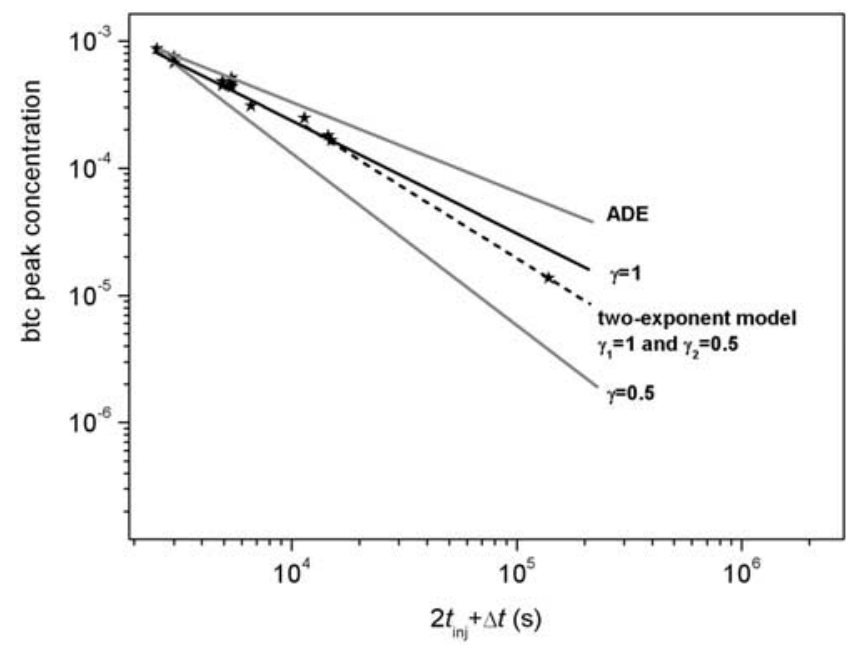

Figure 7. Scaling of the maximum concentration as a function of injection duration (the tracer test data are represented with a star) and expected scaling for the CTRW model, obtained from numerical simulations, with a single exponent $\gamma=1$, with a single exponent $\gamma=0.5$ (double porosity model), and with two exponents $\gamma_{1}=1$ and $\gamma_{2}=$ 0.5 , and for the ADE. 


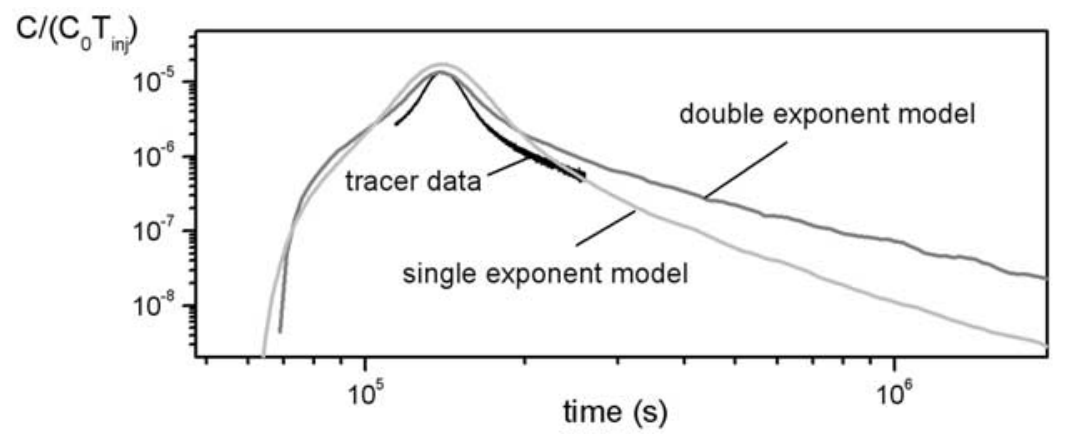

Figure 8. Comparison of tracer data and model predictions (single exponent model with $\gamma=1$ and twoexponent model with $\gamma_{1}=1$ and $\gamma_{2}=0.5$ ) for the long-term conductivity experiment.

maximal tracer penetration exceeded the length of the packers. Thus, part of the tracer may have been irreversibly diluted in the borehole above the upper packer and below the lower packer. Figure 8 shows the prediction of the models fitted on the short-term conductivity BTCs $(\gamma=1)$ and on the uranine BTCs (double-exponent model with $\gamma_{1}=1$ and $\gamma_{2}=0.5$ ). The shape of the single exponent MIM mass transfer model, more specifically the evolution of the late time concentration, is not consistent with the measured BTC. The double-exponent model is found to be consistent with the shape of the BTC (Figure 8), although the width of the central part is slightly overestimated.

[32] We believe that, for the long-term test, the limit of validity of the one-dimensional model is reached. Better estimation would require accounting for true flow field distribution around the packer ends. Nevertheless, it is worth noticing again that the slope of the BTC, after the main peak as passed, is well reproduced and confirm the dual-slope waiting time distribution model. More specifically, we observe that for this push duration, (1) the shape of the BTC is mainly controlled by the MIM mass transfers and (2) the concentration decrease following the concentration peak is mainly controlled by the asymptotic retention regime, i.e., $\gamma_{2}=0.5$. This behavior could not have been predicted from fitting the $\gamma=1$ single retention model with the short-duration tracer test using tracer 1 .

\section{Conclusions}

[33] In first instance, this study showed that the use of high-resolution solute concentration measurement is critical when investigating solute dispersion, in particular when there is evidence of MIM mass transfer processes. MIM mass transfer processes control late time low concentrations for the short-term tracer tests, whereas it is expected that for large-scale displacement, the tracer distribution is dominantly controlled by the MIM mass transfer processes. Then, when making long-term predictions from short-term tracer tests (which is certainly the most probable case), high-resolution measurements are required to tackle the controlling processes.

[34] For the present tracer tests, the fitting of a single breakthrough curve for the low-resolution conductivity tracer tests shows a good fit of the classical MIM mass transfer model with an exponent $\gamma=1$. However, this model does not allow predicting the late time concentration decrease of the BTCs obtained using fluorescent dye high-resolution measurements. The uranine breakthrough curves can be well modeled using a CTRW model with two exponents $\left(\gamma_{1}=1\right.$ for early times and $\gamma_{2}=0.5$ for late times). Such model also allows predicting well the lowresolution conductivity breakthrough curves.

\subsection{Investigation of Heterogeneities at Different Scales for Model Validation}

[35] High-resolution concentration measurement are important but not sufficient in general since the fit of a model using a single BTC curve is not enough for assessing the physical relevance of the transport model. For the considered dispersion model, it is assumed that solutes traveling in the medium are trapped and released from immobile zones. This class of models has been used in several studies to fit breakthrough curves with a power law late time scaling. An important question is whether such model is just convenient to fit BTCs by increasing the number of parameters compared to the classical ADE model, or whether it is really making physical sense. The use of tracer tests that have different volume of investigation provides a way to investigate the physical relevance of the proposed model. Performing SWIW tracer tests with different injection times $\left(T_{\text {push }}\right)$, allows the tracer to visit different volumes of rock before returning to the well, thus investigating possibly different characteristic sizes of heterogeneity.

[36] Thanks to this range of scale investigation, the transport model validation can be done by testing its capacity to reproduce the temporal evolution of the concentration in a single breakthrough curve (Figure 3) and by testing its capacity to predict the amount of mixing as a function of the volume visited by the tracer (Figure 7). The comparison of model prediction and breakthrough curves at different scales shows that over this range of scales, the model fitted at a given scale can predict transport at larger scale. The non-Fickian properties and the scaling of the retention time distribution are found to be independent of the scale. Thus, it appears that for the tested medium, a homogenization scale is reached and the heterogeneity distribution for the larger-scale experiment is consistent with the heterogeneity distribution of the smaller-scale experiment.

\subsection{Physical Meaning of the Model}

[37] The conceptual picture underlying the MIM mass transfer model is the occurrence of immobile zones characterized by a specific range of transfer rates, which can be viewed as a specific range of immobile zone sizes. For the test site, borehole wall images and parameters derived from 
geophysical logging show a relatively homogeneous rock structure at meter scale. Conversely, three-dimensional microtomography images display a heterogeneous pore structure. At this scale, one can identify structures where solute movement is expected to occur mainly by diffusion. These structures include dead ends, disconnected pores and the microporous phase. Their sizes range typically from a few micrometers to less than one millimeter [Gouze et al., 2008]. As a result, the maximum retention time is expected to be short. However, since the surface area for exchanges between mobile and immobile zones is large, tracer molecules may be repeatedly trapped in the immobile zone over a given travel time, so that some tracer molecules take a very long time to move across a given distance.

[38] Above the largest diffusion timescale in the immobile zones, the transport behavior should be expected to be asymptotically Fickian [Dentz et al., 2004]. This timescale is not reached in our experiments. Our results show a transition from anomalous to double-porosity transport. Several physical justifications may be proposed. The first is that the distribution of immobile zone sizes or of the distribution of diffusion coefficients is such that the memory function is not a simple power law function of time. This can be obtained for example if the distribution of immobile zone characteristic sizes is not a power law. A second possible physical justification is that the two power law behaviors are related to different physical processes. For example, the first behavior could be related to the velocity heterogeneity within the pores while the second behavior could be related to diffusive mass transfer into immobile zones. The flow distribution may be characterized by a broad distribution of velocities rather than by a separation between mobile and immobile zones. The relation between the velocity field organization and its correlation structure and the relevant transport model is still an open question [Le Borgne et al., 2007]. Hence, how to investigate the difference between the existence of immobile zones and the existence of a power law distribution of velocities is still an open issue. In an upcoming paper (P. Gouze et al., NonFickian dispersion in porous media explained by heterogeneous microscale matrix diffusion, submitted to Water Resources Research, 2008), we discuss the mass transfer process in the tested rock from numerical simulation of diffusion in the immobile part of the porosity identified from three-dimensional microtomography images. Finally, it must be emphasized that the use of those results for largescale predictive models for pollutant dispersion in this aquifer would require incorporating explicitly large scale heterogeneities, which are not characterized by the SWIW method [Cortis et al., 2004].

[39] Acknowledgments. This work was funded by the European project "ALIANCE" (Advanced Logging Investigations in Coastal Environments, contract EKV-2001-00039). All the members of the ALIANCE team are gratefully acknowledged for their support, especially the coordinator of the project, P. Pezard. Concha Gonzalez and Alfreto Baron, representing the Ministry of Environment of the Balearic Islands Government, are chiefly thanked for their support in developing the Campos experimental site. The authors wish to thank Marco Dentz and Jesus Carrera for numerous stimulating discussions on this topic.

\section{References}

Adams, A. E., and L. W. Gelhar (1992), Field study of dispersion in a heterogeneous aquifer: 2. Spatial moments analysis, Water Resour. Res., 28(12), 3293-3307.
Becker, M. W., and A. M. Shapiro (2003), Interpreting tracer breakthrough tailing from different forced-gradient tracer experiment configurations in fractured bedrock, Water Resour. Res., 39(1), 1024, doi:10.1029/ 2001WR001190.

Benson, D. A., R. Schumer, M. M. Meerschaert, and S. W. Wheatcraft (2001), Fractional dispersion, Lévy motion, and the MADE tracer tests, Transp. Porous Media, 42, 211-240, doi:10.1023/A:1006733002131.

Berkowitz, B., J. Klafter, R. Metzler, and H. Scher (2002), Physical pictures of transport in heterogeneous media: Advection-dispersion, randomwalk, and fractional derivative formulations, Water Resour. Res., 38(10), 1191, doi:10.1029/2001WR001030.

Berkowitz, B., A. Cortis, M. Dentz, and H. Scher (2006), Modeling nonFickian transport in geological formations as a continuous time random walk, Rev. Geophys., 44, RG2003, doi:10.1029/2005RG000178.

Carrera, J., X. Sánchez-Vila, I. Benet, A. Medina, G. Galarza, and J. Gimera (1998), On matrix diffusion: Formulations, solution methods and qualitative effects, Hydrogeol. J., 6(1), 178-190, doi:10.1007/ s100400050143.

Cortis, A., C. Gallo, H. Scher, and B. Berkowitz (2004), Numerical simulation of non-Fickian transport in geological formations with multiple-scale heterogeneities, Water Resour. Res., 40, W04209, doi:10.1029/ 2003WR002750.

Delay, F., P. Ackerer, and C. Danquigny (2005), Simulating solute transport in porous or fractured formations using random walk particle tracking: A review, Vadose Zone J., 4, 360-379, doi:10.2136/vzj2004.0125.

Dentz, M., and B. Berkowitz (2003), Transport behavior of a passive solute in continuous time random walks and multirate mass transfer, Water Resour. Res., 39(5), 1111, doi:10.1029/2001WR001163.

Dentz, M., A. Cortis, H. Scher, and B. Berkowitz (2004), Time behavior of solute transport in heterogeneous media: Transition from anomalous to normal transport, Adv. Water Resour., 27(2), 155-173, doi:10.1016/j. advwatres.2003.11.002.

Gelhar, L. W., and M. A. Collins (1971), General analysis of longitudinal dispersion in nonuniform flow, Water Resour. Res., 7(6), 1511-1521, doi:10.1029/WR007i006p01511.

Gouze, P., T. Le Borgne, R. Leprovost, G. Lods, T. Poidras, and P. Pezard (2008), Non-Fickian dispersion in porous media: 1. Multiscale measurements using single-well injection withdrawal tracer tests, Water Resour. Res., doi:10.1029/2007WR006278, in press.

Haggerty, R., and S. M. Gorelick (1995), Multiple-rate mass transfer for modeling diffusion and surface reactions in media with pore-scale heterogeneity, Water Resour. Res., 31(10), 2383-2400.

Haggerty, R., S. A. McKenna, and L. C. Meigs (2000), On the late-time behavior of tracer test breakthrough curves, Water Resour. Res., 36(12), 3467-3480, doi:10.1029/2000WR900214.

Haggerty, R. S., S. W. Fleming, L. C. Meigs, and S. A. McKenna (2001), Tracer tests in a fractured dolomite: 2. Analysis of mass transfer in single-well injection-withdrawal tests, Water Resour. Res., 37(5), 1129-1142, doi:10.1029/2000WR900334.

Harvey, C. F., and S. M. Gorelick (2000), Rate-limited mass transfer or macrodispersion: Which dominates the plume evolution at the Macrodispersion Experiment (MADE) site?, Water Resour. Res., 36(3), 637650, doi:10.1029/1999WR900247.

Khrapitchev, A. A., and P. T. Callaghan (2003), Reversible and irreversible dispersion in a porous medium, Phys. Fluids, 15, 2649-2660, doi:10.1063/1.1596914.

Kinzelbach, W. (1988), The random walk method in polutant transport simulation, in Ground Water Flow and Quality Modelling, edited by E. Custodio, A. Gurgui, and J. P. Lobo Ferreira, pp. 227-246, Reidel, D., Hinghal, Mass.

Kitanidis, P. K. (1994), The concept of the dilution index, Water Resour. Res., 30(7), 2011-2026, doi:10.1029/94WR00762.

Le Borgne, T., J.-R. de Dreuzy, P. Davy, and O. Bour (2007), Characterization of the velocity field organization in heterogeneous media by conditional correlation, Water Resour. Res., 43, W02419, doi:10.1029/ 2006WR004875.

Levy, M., and B. Berkowitz (2003), Measurement and analysis of nonFickian dispersion in heterogeneous porous media, J. Contam. Hydrol., 64(3-4), 203-226, doi:10.1016/S0169-7722(02)00204-8.

Magnico, P., C. Leroy, J. P. Bouchaud, C. Gauthier, and J. P. Hulin (1993), Tracer dispersion in porous media with a double porosity, Phys. Fluids A, 5, 46-57, doi:10.1063/1.858788.

Meigs, L. C., and R. L. Beauheim (2001), Tracer tests in fractured dolomite: 1. Experimental design and observed tracer recoveries, Water Resour. Res., 37(5), 1113-1128, doi:10.1029/2000WR900335.

Sánchez-Vila, X., and J. Carrera (2004), On the striking similarity between the moments of breakthrough curves for a heterogeneous medium and a 
homogeneous medium with a matrix diffusion term, J. Hydrol., 294(1-3), 164-175.

Scher, H., and M. Lax (1973), Stochastic transport in a disordered solid, I, Theory, Phys. Rev. B, 7(10), 4491-4502, doi:10.1103/PhysRevB.7.4491.

Schumer, R., D. A. Benson, M. M. Meerschaert, and B. Baeumer (2003),

Fractal mobile/immobile solute transport, Water Resour. Res., 39(10), 1296, doi:10.1029/2003WR002141.

Silliman, S. E., and E. S. Simpson (1987), Laboratory evidence of the scale effect in dispersion of solutes in porous media, Water Resour. Res., 23(8), $1667-1673$.

Torquato, S. (2001), Random Heterogeneous Materials: Microstructure and Macroscopic Properties, Interdisciplinary Appl. Math., vol. 16, 701 pp., Springer, New York.
Tsang, Y. W. (1995), Study of alternative tracer tests in characterizing transport in fractured rocks, Geophys. Res. Lett., 22(11), 1421-1424, doi:10.1029/95GL01093.

Tsang, Y. W., and C. F. Tsang (2001), A particle-tracking method for advective transport in fractures with diffusion into finite matrix blocks, Water Resour. Res., 37(3), 831-836, doi:10.1029/2000WR900367.

P. Gouze, Géosciences, UMR 5243, Université de Montpellier 2, CNRS, F-5243, Montpellier, France.

T. Le Borgne, Géosciences Rennes, UMR 6118, Université de Rennes 1, CNRS, F-35042, Rennes, France. (tanguy.le-borgne@univ.rennes1.fr) 